\title{
LARGE-EDDY SIMULATION OF TRANS- AND SUPERCRITICAL INJECTION
}

\section{H. Müller ${ }^{1}$, C. A. Niedermeier ${ }^{2}$, M. Jarczyk ${ }^{1}$, M. Pfitzner ${ }^{1}$, S. Hickel ${ }^{2,3}$, and N. A. Adams ${ }^{2}$}

\author{
${ }^{1}$ Universität der Bundeswehr München \\ Institute for Thermodynamics \\ 39 Werner-Heisenberg-Weg, Neubiberg 85577, Germany \\ ${ }^{2}$ Technische Universität München \\ Institute of Aerodynamics and Fluid Mechanics \\ 15 Boltzmannstr., Garching, Munich 85748, Germany \\ ${ }^{3}$ Faculty of Aerospace Engineering, Technische Universiteit Delft \\ 1 Kluyverweg, Delft 2629 HS, The Netherlands
}

\begin{abstract}
In a joint effort to develop a robust numerical tool for the simulation of injection, mixing, and combustion in liquid rocket engines at high pressure, a real-gas thermodynamics model has been implemented into two computational fluid dynamics (CFD) codes, the density-based INCA and a pressure-based version of OpenFOAM. As a part of the validation process, both codes have been used to perform large-eddy simulations (LES) of trans- and supercritical nitrogen injection. Despite the different code architecture and the different subgrid scale turbulence modeling strategy, both codes yield similar results. The agreement with the available experimental data is good.
\end{abstract}

\section{INTRODUCTION}

In high-performance internal combustion engines as well as in liquid rocket engines (LRE), the propellants may be injected at a super- or transcritical state, i. e., at pressures higher than the critical pressure of the fluid and temperatures close to or below the pseudocritical temperature [1]. The operating pressure in LREs using a gas generator cycle, for instance, lies typically above $10 \mathrm{MPa}$, which is distinctly above the critical pressure of the injected propellants $\left(p_{c, \mathrm{O}_{2}}=5.04 \mathrm{MPa}\right.$ and $\left.p_{c, \mathrm{H}_{2}}=1.4 \mathrm{MPa}\right)$.

In recent years, several groups investigated the physics of cryogenic fluid injection, mixing, and combustion at supercritical pressures. Experiments of a cold supercritical nitrogen jet that is injected into an ambient temperature 
nitrogen environment were conducted in $[2,3]$. Their configuration allows for an isolated view on real-gas dominated mixing processes of a single component flow and is, therefore, well suited to validate numerical methods in this respect. Therefore, these measurements have been selected as a validation database for the numerical tools presented in this paper. Further experiments for inert binary $\mathrm{H}_{2} / \mathrm{N}_{2}$ injection and mixing were presented by Oschwald et al. [4]. Both studies underline that the prevailing physical phenomena change when the operating pressure exceeds the critical point. While subcritical jet-breakup is controlled by droplet formation and evaporation, no phase interface could be observed at supercritical pressures. Combustion of $\mathrm{H}_{2} / \mathrm{O}_{2}$ flames at elevated pressures was studied by Singla et al. [5] as well as by Mayer and Tamura [6]. A comprehensive review on the experimental activities in this field is given by Oschwald et al. [1] and recently by Chehroudi [7].

In addition to these experiments, supercritical fluids have been subject of many numerical studies. Injection, mixing, and combustion at supercritical pressures are strongly influenced by intermolecular forces which are usually neglected at lower pressures (ideal gas assumption). These real-gas effects need to be modeled accurately, which is a serious challenge. An elaborate approach was, for instance, presented by the group of J. Bellan [8-10] who studied shear layers in a supercritical environment using both direct numerical simulation (DNS) and LES. Remarkable progress has also been made in modeling inert as well as reacting jet configurations at supercritical pressures by means of LES [11-15]. These studies mostly used density-based numerical methods, which show a good performance for high-velocity flows. However, near the incompressible limit, the set of governing equations becomes very stiff. This problem does not occur in pressure-based solution methods, which solve an equation for pressure instead of the continuity equation. Recently, pressure-based solution methods have also been applied to supercritical jet configurations [16-19]. While stiffness is not an issue for such codes, stability issues have been reported and modifications to the pressure equation as well as to the solution algorithm are necessary. The stability suffers mainly from the presence of steep density gradients, which are a characteristic of fluids close to the critical density.

In this context, the Technische Universität München (TUM) and the Universität der Bundeswehr München (UniBW) started a joint effort to develop numerical tools that are capable of predicting supercritical mixing and combustion. While the TUM promotes INCA* (density-based), the UniBW extends the capabilities of the open-source CFD program OpenFOAM ${ }^{\dagger}$ (pressure-based). The two LES CFD codes have been extended using the same models of real-gas thermodynamic effects but, otherwise, are developed independently. The cu-

\footnotetext{
*www.inca-cfd.org.

†www.openfoam.com.
} 
bic Peng-Robinson (PR) equation of state (EoS) [20] has been used to describe the thermodynamic behavior of the fluid. As a part of the validation, both codes have already been successfully used to reproduce DNS results of Okong'o et al. [21] for an inert, transitional, temporal mixing layer [22]. OpenFOAM has also been tested for trans- and supercritical nitrogen jets [17] and reproduced the experiments in good agreement. In the following, the numerical method used in both codes as well as the thermodynamic modeling are presented. The procedures are then applied to simulate trans- and supercritical nitrogen jet flows that have been studied experimentally in $[2,3]$. The two codes are compared with respect to modeling accuracy. Furthermore, the sensitivity of the results on the EoS as well as on changes in the temperature boundary condition is discussed.

\section{NUMERICAL AND PHYSICAL MODELING}

\subsection{Governing Equations}

The general compressible conservation equations for mass, momentum, and energy for a single component flow are:

$$
\begin{aligned}
\frac{\partial \rho}{\partial t}+\frac{\partial\left(\rho u_{i}\right)}{\partial x_{i}} & =0 \\
\frac{\partial\left(\rho u_{i}\right)}{\partial t}+\frac{\partial\left(\rho u_{i} u_{j}\right)}{\partial x_{j}} & =-\frac{\partial p}{\partial x_{i}}+\frac{\partial \tau_{i j}}{\partial x_{j}} \\
\frac{\partial\left(\rho e_{t}\right)}{\partial t}+\frac{\partial\left(\rho u_{i} e_{t}\right)}{\partial x_{i}} & =-\frac{\partial\left(u_{i} p\right)}{\partial x_{i}}-\frac{\partial q_{i}}{\partial x_{i}}+\frac{\partial\left(u_{i} \tau_{i j}\right)}{\partial x_{i}} .
\end{aligned}
$$

Here, $x_{i}$ are the Cartesian coordinates; $t$ is the time; $\rho$ is the density; $u_{i}$ is the velocity component in direction $i ; e_{t}$ is the total energy; and $p$ is the thermodynamic pressure. The viscous stress tensor and the heat flux are:

$$
\begin{aligned}
\tau_{i j} & =\mu\left(\frac{\partial u_{i}}{\partial x_{j}}+\frac{\partial u_{j}}{\partial x_{i}}-\frac{2}{3} \frac{\partial u_{k}}{\partial x_{k}} \delta_{i j}\right) ; \\
q_{i} & =-\lambda \frac{\partial T}{\partial x_{i}} .
\end{aligned}
$$

In LES, only turbulent motions with length scales greater than a given filter width are resolved while the subgrid scale (SGS) turbulence has to be modeled. Here, different strategies are employed for the two CFD codes, which are described in the respective sections. The thermophysical properties in the above equations, such as thermal conductivity $\lambda$, viscosity $\mu$, temperature $T$, and pressure $p$, are related to the transported quantities consistently in both codes using the same thermophysical real-gas models. 


\subsection{Thermophysical Modeling}

The cubic PR EoS [20] has been chosen to describe the relation between the thermodynamic state variables:

$$
p=\frac{R T}{v-b}-\frac{a}{v^{2}+2 v b-b^{2}} .
$$

The molar volume is denoted by $v$ and $R$ is the molar gas constant. Intermolecular attractive forces are described by the temperature-dependent function $a$ and the reduction of free volume due to the finite volume of the molecules is taken into account by $b$. Both functions are empirical and normalized to the critical point $\left(T_{c}, p_{c}\right)$ of the fluid they describe:

$$
\begin{aligned}
& a=0.457 \frac{R^{2} T_{c}^{2}}{p_{c}}\left(1-\kappa\left(1-\sqrt{\frac{T}{T_{c}}}\right)\right)^{2} ; \\
& b=0.0778 \frac{R T_{c}}{p_{c}} .
\end{aligned}
$$

The function $\kappa=0.37464+1.54226 \omega-0.26992 \omega^{2}$ accounts for the deviation of the molecules from the perfect circular shape that can be assumed only for monatomic elements, $\omega$ denotes the constant acentric factor $\left(\omega_{\mathrm{N}_{2}}=0.037\right)$. The PR EoS gives accurate predictions for temperatures above and close to the critical temperature; however, in the transcritical regime, distinct deviations from experimental data can be observed. As a remedy to this problem, Matheis et al. [23] evaluated various volume-translation methods for cubic EoSs. By translating the thermodynamic state of the fluid along its volume axis, the density prediction is considerably improved. An example is the empirical method of Harstad et al. [24], which has been implemented in both codes. This volume correction method will henceforth be referred to as PR-VC. To close the set of conservation equations, the caloric properties have to be calculated using the departure function formalism (see also [25]). This method is based on a decomposition of the caloric property into an ideal contribution, which is calculated at a low reference pressure, and a departure function taking into account the pressure effects, e.g., for enthalpy:

$$
h(T, p)=h_{0}(T)+\int_{p_{0}}^{p}\left(V-T\left(\frac{\partial V}{\partial T}\right)_{p}\right) d p
$$

where the subscript 0 refers to the ideal reference state $\left(p_{0}=1\right.$ bar), which is evaluated using the 9-coefficient NASA-polynomials proposed by Goos et al. [26]. The integral on the right side of Eq. (6) is the departure function and needs to 
be calculated using an EoS. The transport properties, i. e., the viscosity and the thermal conductivity, have been modeled with the empirical correlation for dense fluids of Chung et al. [27].

\subsection{INCA Code}

INCA directly solves the compressible Navier-Stokes equations in conservation form (1)-(3) with the Adaptive Local Deconvolution Method (ALDM), which follows an implicit LES (ILES) approach. The basic idea of ILES is to combine turbulence modeling and numerical discretization of the conservation equations. ALDM is a nonlinear finite volume method and incorporates free parameters in the discretization scheme, which can be used to control the truncation error. A physically motivated implicit SGS model that is consistent with turbulence theory is obtained through parameter calibration [28]. ALDM is implemented for Cartesian collocated grids and used to discretize the convective terms of the Navier-Stokes equations. The diffusive terms are discretized by secondorder centered differences and a third-order explicit Runge-Kutta method is used for time integration. For a detailed description of the numerical method, refer to $[29,30]$.

As INCA is a density-based code, pressure and temperature have to be calculated at each time step from internal energy and density. For ideal gases, this can be done in a straightforward manner by using the ideal gas law. However, for real gases, an iterative procedure is necessary to find the correct values for pressure and temperature. A nonlinear least squares optimization method is best suited to efficiently solve this problem. For all simulations presented in this paper, a trust region method is used for the iterative calculation.

\subsection{OpenFOAM Code}

Instead of solving the energy conservation equation (3) directly, an enthalpy equation is used in the OpenFOAM code:

$$
\frac{\partial(\rho h)}{\partial t}+\frac{\partial\left(\rho u_{i} h\right)}{\partial x_{i}}=-\frac{\partial q_{i}}{\partial x_{i}}+\frac{D p}{D t} .
$$

Hereby, the viscous heating term $\partial u_{i} \tau_{i j} / \partial x_{j}$ is not considered, since it is negligible for low-velocity flows. In the LES context, a filtering operation is applied before the governing equations are solved. However, the filtering introduces unresolved turbulent motions, which, in the OpenFOAM code, are modeled with an eddy viscosity approach. Hence, the viscosity $\mu$ and the thermal conductivity $\lambda$ in Eqs. (4) and (5) are incremented by an SGS contribution that is modeled using the explicit Smagorinsky model [31]: 


$$
\begin{aligned}
\mu_{\mathrm{SGS}} & =C_{s}^{2} \Delta^{2} \sqrt{\widetilde{S}_{i j} \widetilde{S}_{i j}} ; \\
\lambda_{\mathrm{SGS}} & =c_{p} \frac{\mu_{\mathrm{SGS}}}{\operatorname{Pr}_{t}}
\end{aligned}
$$

where $C_{s}$ is the Smagorinsky constant, which has been set to 0.17 , and the turbulent Prandtl number $\operatorname{Pr}_{t}$ has been assumed to be $1.0 ; \Delta$ and $\widetilde{S}_{i j}$ denote the filter width and the filtered strain-rate-tensor, respectively:

$$
\widetilde{S}_{i j}=\frac{1}{2}\left(\frac{\partial \widetilde{u_{i}}}{\partial x_{j}}+\frac{\partial \widetilde{u_{j}}}{\partial x_{i}}\right) .
$$

Most CFD simulations for flows with real-gas thermodynamic effects have been performed with density-based codes. However, the OpenFOAM solver pursues a different strategy by using a Pressure Implicit with Splitting of Operators (PISO) algorithm. Originally designed for incompressible flows, the PISO algorithm has been extended to allow for simulations of flows with moderate compressibility effects. Instead of solving for the continuity Eq. (1), an equation for pressure is solved:

$$
\frac{\partial\left(\rho_{0}-\psi_{0} p_{0}\right)}{\partial t}+\frac{\partial \psi_{0} p}{\partial t}+\nabla\left(\rho \frac{H_{p}}{A_{p}}\right)-\nabla\left(\frac{\rho}{A_{p}} \nabla p^{*}\right)=0 .
$$

Here, the subscript 0 refers to the base point of the Taylor expansion, which coincides with the corresponding values of the previous iteration; $\psi=\partial \rho / \partial p$ denotes the compressibility of the fluid; $A_{p}$ is the coefficient matrix of the discretized momentum equation; and $H_{p}$ is the operator including the discretized source term. This pressure equation can be derived from the discretized momentum equation and the continuity equation as shown in detail by Issa et al. $[32,33]$. However, due to the nonlinearity of the PR EoS, it becomes necessary to modify the standard formulation of the pressure equation as well as the segregated solution algorithm, which both imply ideal gas behavior. Jarczyk and Pfitzner [17] have derived an adapted pressure equation using a Taylor expansion to linearize the EoS. The first term in Eq. (7) is zero for ideal gases and, therefore, usually neglected. However, careful modeling of this term is required if real-gas effects are to be considered. A further modification has to be made to the segregated solution algorithm shown in Fig. 1. Since the compressibility in the pressure equation does not depend on the pressure for ideal gases, it is sufficient to evaluate the thermodynamic properties before the PISO loop. However, for a cubic EoS, $\psi$ is a function of pressure and thus has to be updated during the PISO loop in order to achieve convergence. The extended PISO loop is shown in Fig. $1 b$. Both the thermodynamic properties as well as the enthalpy equation are solved along with the pressure equation. This modification clearly increases the computational cost but is necessarily required for convergence and stability. For more 


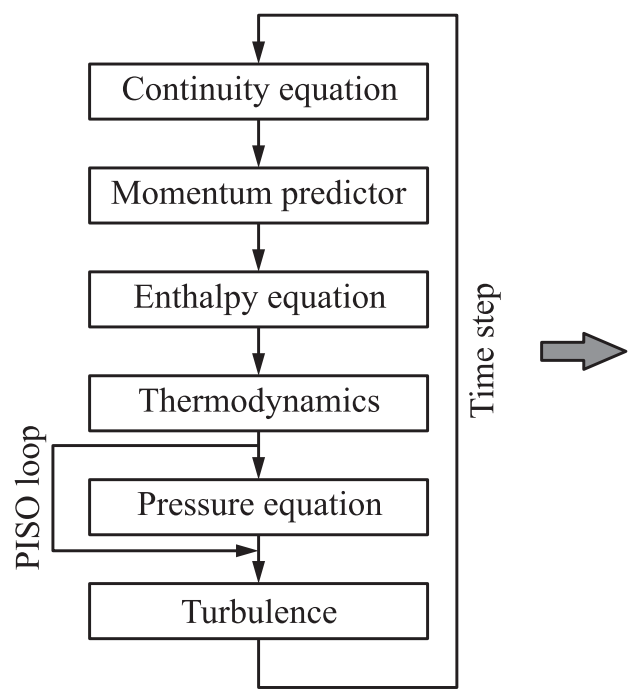

(a)

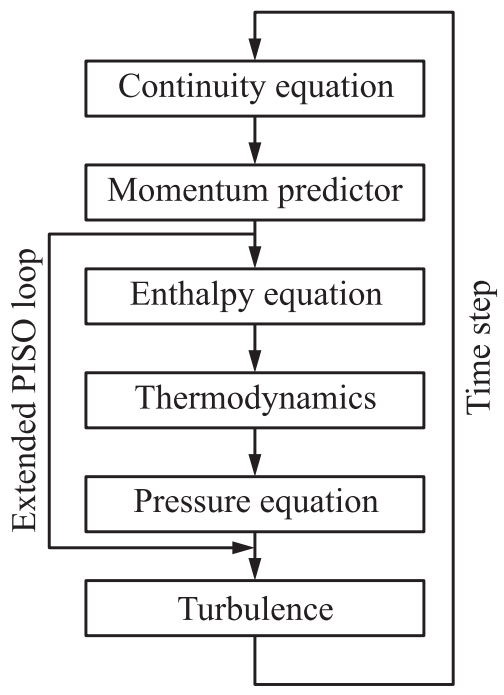

(b)

Figure 1 Segregated, pressure-based PISO solution algorithm for ideal (a) and real gases $(b)$

details on the extended PISO algorithm and the modified pressure equation, refer [17].

All OpenFOAM simulations have been performed using a second-order centered differencing scheme for the spatial discretization. A van Leer limiter is used to avoid unphysical oscillations and a first-order implicit Euler scheme has been used for time integration.

\section{EXPERIMENTAL AND NUMERICAL SETUP}

As a test case for the presented numerical and physical modeling of trans- and supercritical flows, a round jet configuration is chosen. Cold nitrogen is injected into a mixing chamber that is filled with gaseous nitrogen at ambient temperature. This case has been investigated experimentally in $[2,3]$ using a twodimensional (2D) Raman imaging technique. The results allow for a validation of the averaged density profiles obtained from the CFD.

\subsection{Test Cases}

Mayer et al. have conducted a series of experiments covering pressures from 3.96 to $5.98 \mathrm{MPa}$, temperatures from 126.2 to $140.5 \mathrm{~K}$, and injection velocities 
Table 1 Initial and boundary conditions for trans- and supercritical jet flows

\begin{tabular}{ccccc}
\hline $\begin{array}{c}\text { Investigated } \\
\text { cases }\end{array}$ & $\begin{array}{c}\text { Bulk } \\
\text { injection } \\
\text { velocity, } \\
\mathrm{m} / \mathrm{s}\end{array}$ & $\begin{array}{c}\text { Injection } \\
\text { temperature, } \\
\mathrm{K}\end{array}$ & $\begin{array}{c}\text { Chamber } \\
\text { pressure, } \\
\mathrm{MPa}\end{array}$ & $\begin{array}{c}\text { Chamber } \\
\text { temperature, } \\
\mathrm{K}\end{array}$ \\
\hline Case 3 & 4.9 & 126.9 & 3.97 & 298 \\
Case 4 & 5.4 & 137.0 & 3.98 & 298 \\
Case 7 & 4.5 & 126.2 & 5.01 & 298 \\
\hline
\end{tabular}

from 1.8 to $5.4 \mathrm{~m} / \mathrm{s}$. From this database, three configurations, which are summarized in Table 1, have been chosen for validating the new solvers. The nomenclature of the investigated configurations is adapted from the experimental numbering in [3]. Cases 3 and 4 have a similar chamber pressure of 3.97 and $3.98 \mathrm{MPa}$, respectively. This is rather close to the critical pressure of nitrogen $\left(p_{c, \mathrm{~N}_{2}}\right.$ $=3.4 \mathrm{MPa}$ ). The temperature dependence of the thermodynamic properties, such as heat capacity and density, around the pseudocritical temperature is, therefore, very pronounced. Figure $2 a$ (left column) shows the density curve at the operating pressure of Cases 3 and 4 as well as the inlet conditions of the two cases. While the measured inlet temperature of Case $3\left(T_{\mathrm{inj}}=126.9 \mathrm{~K}\right)$ lies below the pseudocritical temperature, the inlet temperature of Case $4\left(T_{\mathrm{inj}}=137 \mathrm{~K}\right)$ is supercritical. From a modeling point of view, Case 3 is the most interesting, since the regime of sharp density gradients has to be resolved during the mixing and heating of the cold jet. The influence of the thermodynamic modeling is expected to exert the greatest influence on the jet compared to the other operating conditions considered in the current work. For Case 4, the regime of large density gradients is not passed and thermodynamic modeling is, therefore, less critical.

The same holds for the isobaric heat capacity which is shown in Fig. $2 b$ (left column). While the peak in heat capacity at the pseudocritical temperature has to be passed for transcritical injection temperatures (Case 3), it is avoided at higher injection temperatures (Case 4).

Also, in Fig. 2, different EoSs are compared in order to assess their suitability for the particular configurations considered in the present study. The PR EoS, both with and without the volume correction method, as well as the often utilized Soave-Redlich-Kwong (SRK) EoS [35] are compared with NIST data [34], which serve as a reference. As anticipated, Case 3 is the most critical and shows distinct differences between the models. The advantage of the volume correction method lies in the regime of cryogenic temperature, where the uncorrected PR EoS is known to significantly overpredict the density. However, for this particular configuration, the uncorrected PR EoS is actually better suited than the 

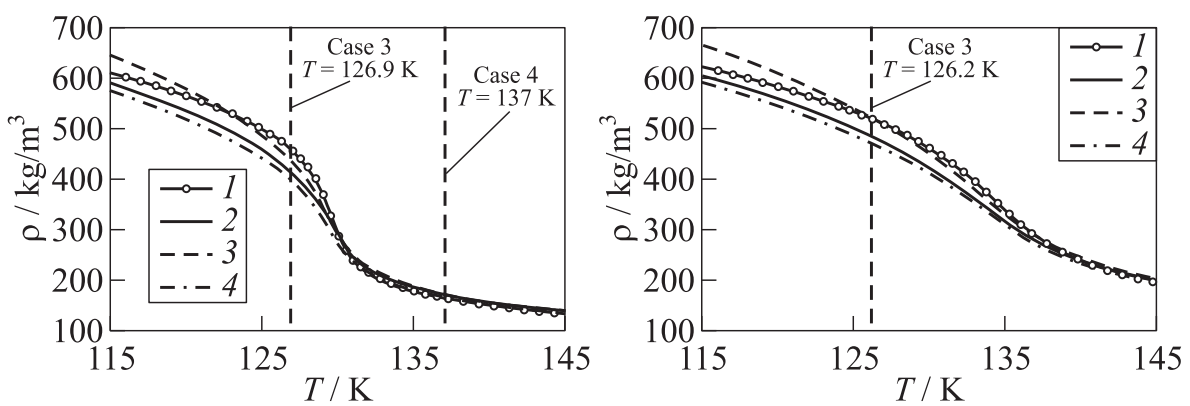

(a)
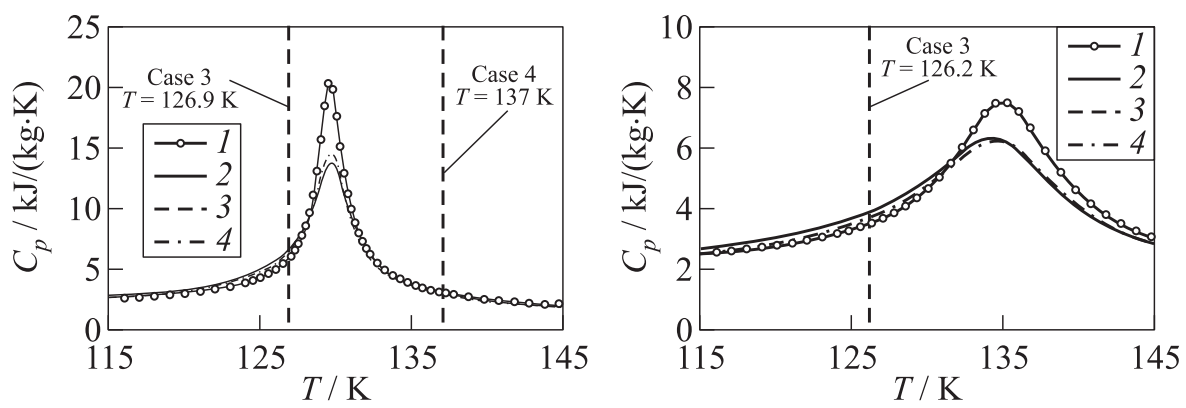

(b)

Figure 2 Density $(a)$ and isobaric heat capacity $(b)$ as obtained with different EoS in comparison with NIST [34] reference data at $p=3.97$ (left column) and $5.01 \mathrm{MPa}$ (right column): 1 - NIST; $2-\mathrm{PR}-\mathrm{VC} ; 3-\mathrm{PR}$; and $4-\mathrm{SRK}$

PR-VC EoS. For the sake of future simulations, in which the operating points are in regimes that are better represented by the volume correction method, the two codes are compared using the PR-VC EoS. The SRK EoS does not perform particularly well and is, therefore, not used for the LES in this study. Case 7 features approximately the same injection temperature as Case 3 but a higher chamber pressure of 5.01 MPa. The density and heat capacity curves for this operating point, calculated with different EoSs, are shown in Figs. $2 a$ and $2 b$ (right column). Like for Case 3 , the measured inlet temperature $\left(T_{\mathrm{inj}}=126.2 \mathrm{~K}\right)$ lies below the pseudocritical temperature. Thus, both the heat capacity peak and the steep density gradient are passed during heating of the dense core. However, the temperature sensitivity of density as well as of heat capacity is less critical than for Case 3 as the pressure is farther away from the critical pressure $p_{c, \mathrm{~N}_{2}}$. A comparison of the accuracy of different EoS shows the same trend previously observed for lower pressure, i. e., the uncorrected PR EoS shows the best agreement with the NIST data. 


\subsection{Computational Setup}

The experimental injector has a radius of $R=1.1 \mathrm{~mm}$ and is long enough to ensure fully turbulent flow conditions at the injector exit. The testing chamber is a cylindrical tank $\left(D_{c}=122 \mathrm{~mm}\right)$ and is initially filled with nitrogen at ambient temperature. While the chamber front wall has been assumed to be adiabatic, the outer walls have been kept at a constant temperature of $298 \mathrm{~K}$. More details on the experimental setup are given in [36].

For the INCA simulations, a block-structured Cartesian grid with about 3.9 million cells was used. The outer dimensions of the domain are $60 \times 60 \times 80 \mathrm{~mm}$. The grid is refined near the centerline whereas the axial grid spacing is kept homogeneous. At the inflow, a fully turbulent mean velocity profile was prescribed as obtained from the one-seventh power law for pipe flow [37]. Low-intensity white noise fluctuations are superimposed to model turbulent conditions.

In the OpenFOAM simulations, a cylindrical grid was used. The diameter of the domain coincides with the experimental setup and comprises 1.7 million cells. The grid is stretched in axial as well as in radial directions and is additionally refined near the shear layer where warm and cold nitrogen mix. The transient velocity field at the inflow is generated with a separate incompressible LES using cyclic boundary conditions in axial direction. A validation of the method can be found in [38]. From the pipe flow, the slices of the turbulent velocity field were extracted, which are then interpolated onto the inflow boundary of the jet simulation. In order to examine the sensitivity of the results on the resolution, the simulations were also performed using a grid with 4.1 and 7.2 million cells.

\section{RESULTS}

\subsection{Instantaneous Results}

In Fig. $3 a$, snapshots of the density gradient magnitudes are shown. The isolines refer to the arithmetic mean density between the injector exit and the surrounding nitrogen and illustrate the jet breakup. The two cases with injection temperatures below the pseudocritical temperature (Cases 3 and 7) are characterized by a sharp density gradient that encloses the cold jet. The large density ratio between the core and the surrounding hinders the formation of Kelvin-Helmholtz vortices [39] and, therefore, delays the jet breakup. Zong et al. [40] argued that in this area, strong local anisotropies transfer turbulence kinetic energy from a direction perpendicular to the jet surface to the streamwise direction. This wall-like behavior is also reflected in Fig. 4 where the isocontours of the arithmetic mean density are shown for Cases 3 and 4 . It can be observed that the structure of the jet surface downstream of the injector $(x / R<5)$ differs between 

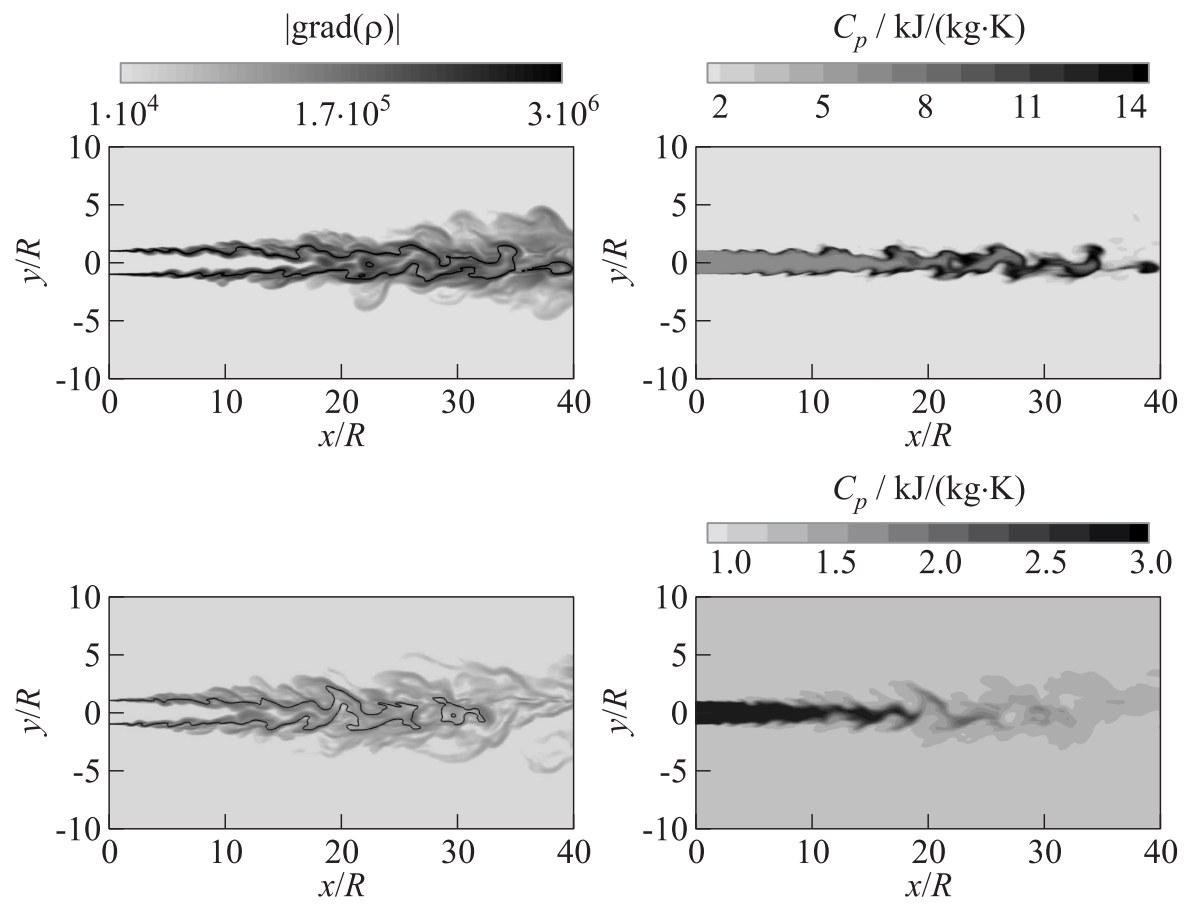

$$
C_{p} / \mathrm{kJ} /(\mathrm{kg} \cdot \mathrm{K})
$$

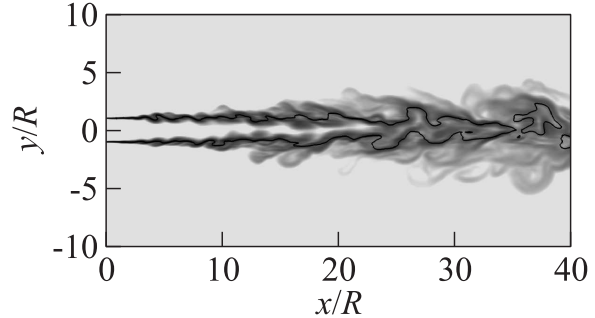

(a)

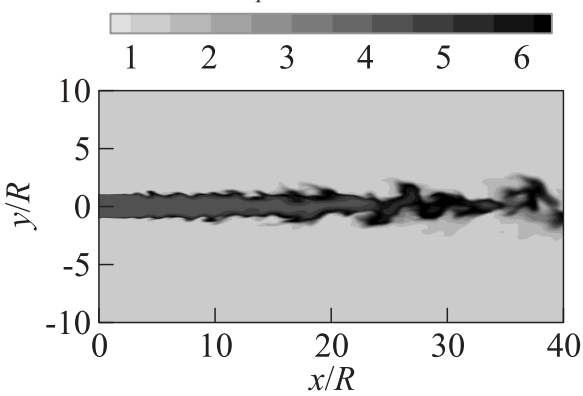

(b)

Figure 3 Magnitude of the density gradients (black isoline denotes the arithmetic mean density between inlet and ambient nitrogen) $(a)$ and isobaric heat capacity contour plots (b) for Cases 3 (top), 4 (middle), and 7 (bottom) (OpenFOAM) 


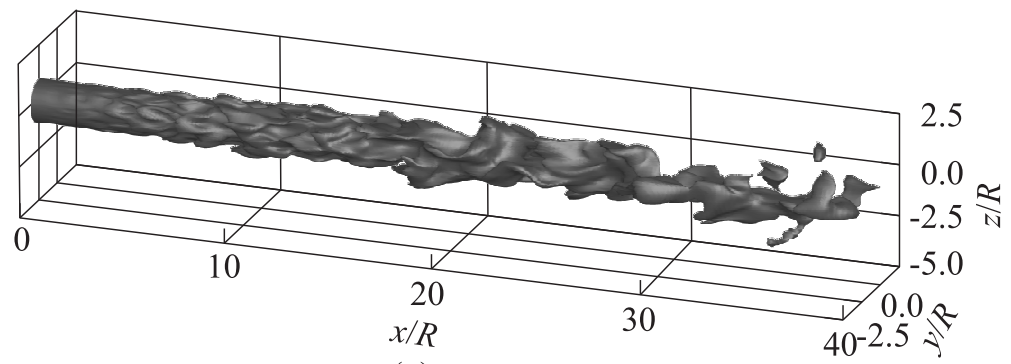

(a)

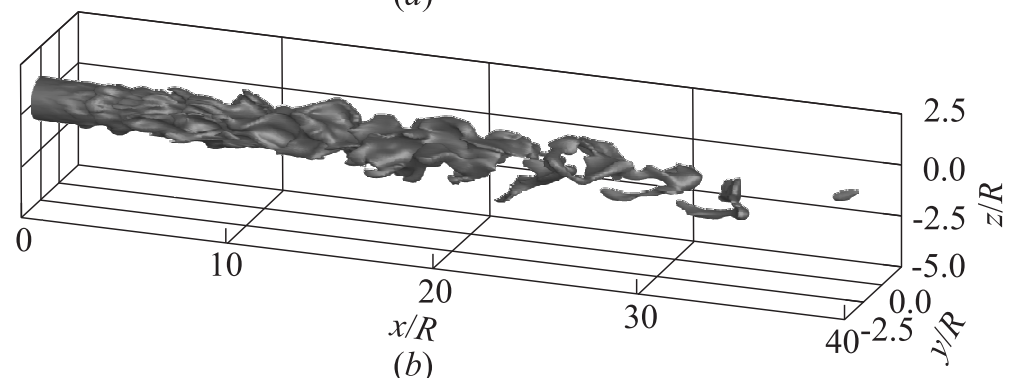

Figure 4 Instantaneous isocontours of density. The threshold is the arithmetic mean between chamber and inflow condition $\rho_{\text {iso }}=0.5\left(\rho_{\text {inj }}+\rho_{\infty}\right):(a)$ Case 3, $\rho_{\text {iso }}=221.5 \mathrm{~kg} / \mathrm{m}^{3} ;$ and $(b)$ Case $4, \rho_{\text {iso }}=109.5 \mathrm{~kg} / \mathrm{m}^{3}$

the two cases. In Case 4, coherent vortices evolve significantly closer to the injector than in Case 3 where the damping of the radial fluctuations is stronger. In Fig. 3b, the corresponding instantaneous isobaric heat capacity is shown. At the shear layer between warm and cold nitrogen of Cases 3 and 7, a layer of high heat capacity can be observed. This layer corresponds to the heat capacity peak at the pseudocritical temperature previously mentioned (see Fig. 2). In these transcritical cases, a higher amount of enthalpy is needed to obtain a certain temperature increment at the surface of the cryogenic core. As a result, the steep density gradient at the shear layer is more resistant to heat conduction from the warm surrounding. This additionally stabilizes Cases 3 and 7 in comparison to Case 4 where the injection temperature is higher than the pseudocritical temperature and no layer of high heat capacity can be observed.

\subsection{Axial Density Profiles}

Figure 5 shows the time-averaged density profiles of both codes in comparison with each other and with the experimental data. Despite the various differences between INCA and OpenFOAM, the results for both codes are in close agree- 


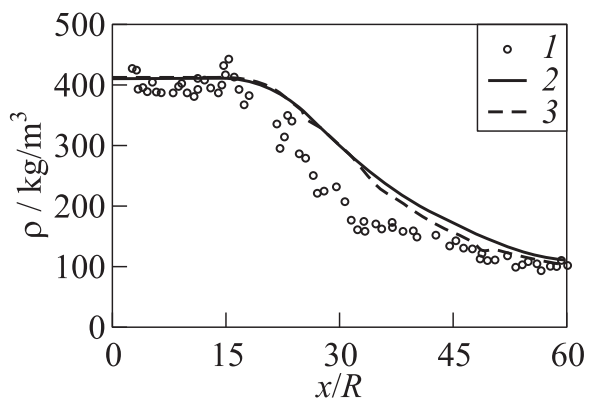

(a)

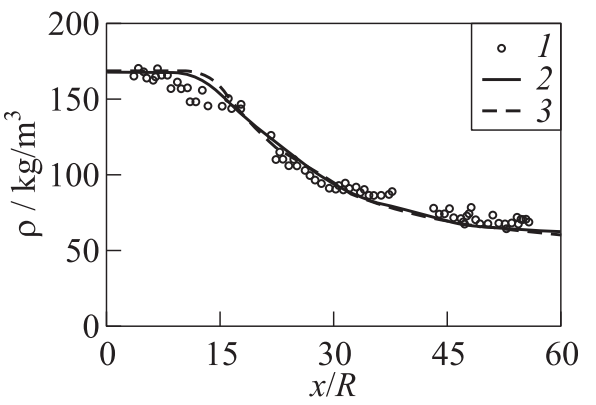

(b)

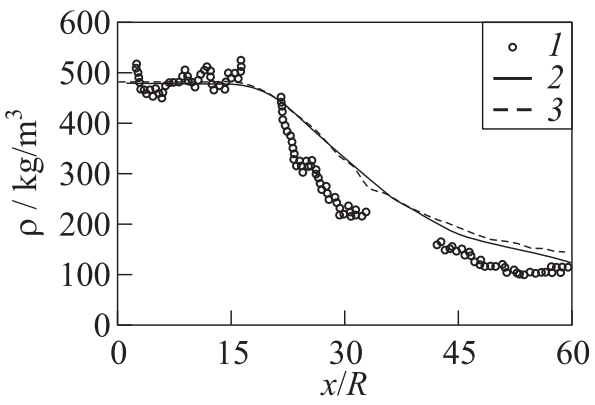

(c)

Figure 5 Mean density profiles on the centerline for Cases $3(a), 4(b)$, and $7(c)$ : 1 - experiments [3]; 2 - OpenFOAM; and 3 - INCA

ment. The maximum deviation between the results for Case 3 can be found at $x / R \approx 45$ and amounts to approximately $9.2 \%$ with the INCA result as a reference. In comparison, the inherent modeling error of the SRK and PR-VC EoS with respect to the NIST chemistry webbook [34] (see Fig. 2) at the injector exit is $12.3 \%$ and $10.2 \%$, respectively. This is remarkable, since the codes differ in several aspects, such as SGS modeling, mesh resolution, the method to generate the turbulent inflow condition, discretization, and code architecture. Nevertheless, with no particular adjustments made, the deviation in the axial mean density is smaller than the inherent modeling error of the EoS. Moreover, the size of the simulated domain differs between the OpenFOAM and the INCA simulations. While for INCA, a rectangular duct is used with an edge length of $60 \mathrm{~mm}$, for OpenFOAM, a cylindrical domain with the diameter $122 \mathrm{~mm}$ is chosen. It appears that the smaller domain is sufficient and the development of the jet is not influenced by the outer boundary.

The measurements of Mayer et al. [3] serve as a basis to validate the described simulation results. All three cases are reproduced in satisfactorily agreement 
with the experiments. Deviations can be observed in the region of jet breakup $(20<x / R<40)$ for the transcritical configurations (Cases 3 and 7 ), where the steep density decrease is not fully captured. The simulations for the supercritical Case 4 match the experiments particularly well.

\subsection{Influence of the Equation of State}

As mentioned before, the uncorrected PR EoS is expected to be better suited for the configurations considered in this study. Especially for the transcritical conditions in Cases 3 and 7, the predicted density matches the NIST data better than the PR-VC or the SRK EoS (see Fig. 2a). For this reason, further LES have been carried out with the PR EoS, while leaving all other models unchanged. Case 3 has been chosen for this study, since it is the most sensitive configuration in terms of thermodynamic modeling. Figure 6 shows the mean density as obtained with OpenFOAM for both the PR-VC EoS (curve 2) and the PR EoS (curve 3). Compared to the differences that were observed between the two codes in the previous section (see Fig. $5 a$ ), the differences caused by changing the EoS are distinctly more pronounced. This can be attributed directly to the differences in the EoS only in the section prior to jet breakup $(x / R<20)$. Further downstream, the temperature on the centerline increases and the difference between the uncorrected and the corrected PR EoS becomes negligible (see Fig. $2 a$, left column). However, the difference between the two density curves obtained from the simulation persists over the whole range, which shows that the choice of the EoS affects the mixing process.

It may appear contradictory that the result obtained with the PR EoS is in worse agreement with the experiment than the result obtained with the PR-VC EoS even though it performs better with respect to the NIST data. However, the authors of $[3,36]$ discuss that despite using sophisticated methods, they had problems to determine the exact temperature at the nitrogen inflow. Also, the measurement data were recorded in a transient phase in which the feeding lines did not have the same temperature as the injected fluid. It is probably due to a combination of these issues that the measured density in the first section after the injector is approximately $\Delta \rho=60 \mathrm{~kg} / \mathrm{m}^{3}$ smaller than the density that can be obtained from NIST [34] using the measured inlet temperature and chamber pressure. This inconsistency at the boundary explains why the result obtained using the PR-VC EoS, which also underpredicts the density with respect to NIST, is closer to the experiment.

\subsection{Influence of the Inlet Temperature}

Another simulation has been performed using a modified value for the inflow temperature in order to better assess the suitability of the EoS and to investigate 


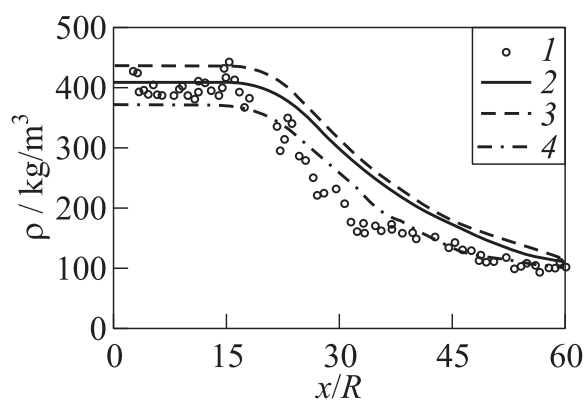

Figure 6 Mean density on the centerline for Case 3 with different EoSs and inlet temperatures (OpenFOAM): $1-$ experiments $[3] ; 2-$ PR-VC EoS, $T_{\text {in }}=126.9 \mathrm{~K}$; $3-\mathrm{PR} \operatorname{EoS}, T_{\mathrm{in}}=126.9 \mathrm{~K}$; and $4-\mathrm{PR} \operatorname{EoS}, T_{\mathrm{in}}=128.53 \mathrm{~K}$

the influence of inflow temperature variations on the result. Previous numerical studies demonstrate that small temperature changes may have a significant impact on the centerline density. Among them is the work of Mayer et al. [3] who perform computations with the maximum and the minimum temperature values that were obtained in the experiments, thereby covering a range of $\Delta T=4 \mathrm{~K}$ for Case 3. Banuti and Hannemann [41] additionally account for the heat addition in the injector tube. The present authors pursue an alternative approach and calculate the inflow temperature taking the density measurements close to the injector as a reference. By averaging the experimental datapoints in the section prior to jet breakup, an experimental mean density at the injector exit of $\bar{\rho}_{\text {inj }}=398 \mathrm{~kg} / \mathrm{m}^{3}$ can be estimated. With a highly accurate EoS, as available in the NIST chemistry webbook [34], the injection temperature for Case 3 can then be calculated as $\bar{T}_{\text {inj }} \approx 128.53 \mathrm{~K}$. Though the difference to the measured value is only $\Delta T=1.63 \mathrm{~K}$, it implies a density variation of $\Delta \rho \approx 60 \mathrm{~kg} / \mathrm{m}^{3}$. The results obtained with the modified injection temperature are shown in Fig. 6 (curve 4). It was found that modifying the inlet temperature as described has the effect to shift the density curve to smaller values without significantly changing its overall slope. Even though the discrepancy between experiment and LES is now greater than in the simulation using the PR-VC EoS (curve 2), using the modified inflow temperature allows for an isolated assessment of the thermodynamic models. The discrepancy in the section prior to jet breakup $(x / R<20)$ is now entirely due to the inherent modeling error of the EoS and misleading agreement between simulation and experiment is thus avoided.

\subsection{Grid Convergence Study}

In order to evaluate the influence of the numerical resolution, two additional simulations have been carried out with the OpenFOAM code using refined grids. 


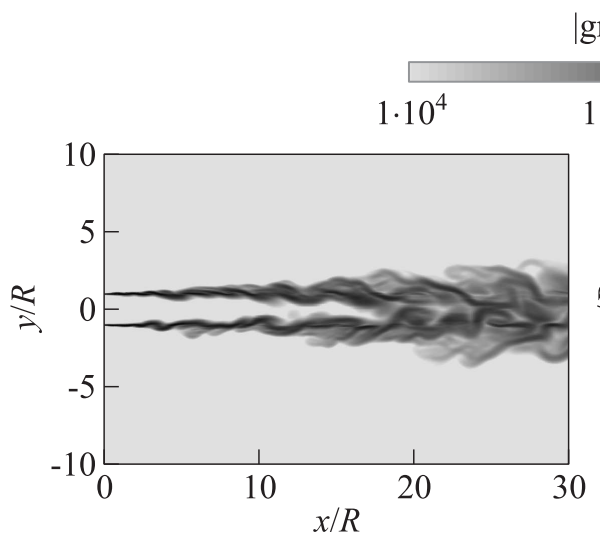

(a) $|\operatorname{grad}(\rho)|$

$1.7 \cdot 10^{5} \quad 3 \cdot 10^{6}$

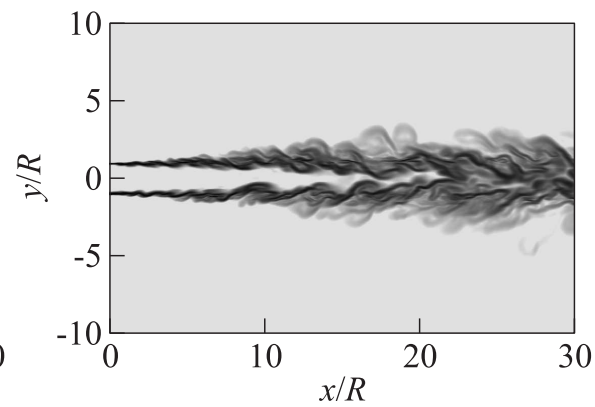

(b)

Figure 7 Instantaneous density gradient magnitudes for Case 3 with different mesh resolutions as obtained with OpenFOAM: $(a) 1.7 \cdot 10^{6}$ cells; and (b) $7.2 \cdot 10^{6}$ cells

For the first simulation, the grid is refined by a factor of 1.35 in each direction and comprises 4.1 million cells. For the second simulation, 7.2 million grid cells have been used. The simulations have been performed for Case 3 with the PR EoS and the original inlet temperature as measured by Mayer et al. [3]. The instantaneous density gradient magnitudes for the coarse and the fine grid are compared in Fig. 7. While the turbulent fluctuations are clearly better resolved on the fine grid, the breakup angle and the shape of the jet appears to be similar. This is underlined by the mean density along the centerline shown in Fig. 8. Although the location of jet breakup moves slightly upstream in the

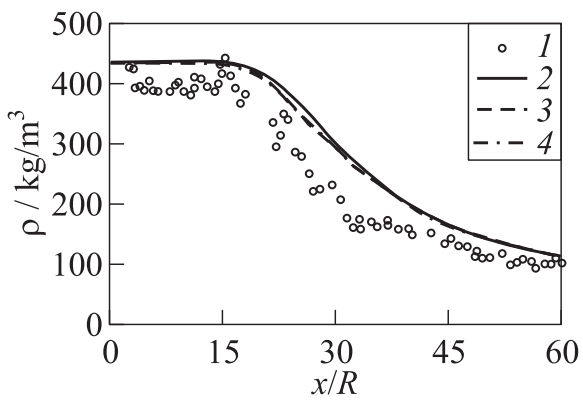

Figure 8 Mean density profiles for Case 3 with different mesh resolutions as obtained with OpenFOAM: 1 - experiments $[3] ; 2-$ coarse $\left(1.7 \cdot 10^{6}\right.$ cells $) ; 3-$ medium $\left(4.1 \cdot 10^{6}\right.$ cells $)$; and $4-$ fine $\left(7.2 \cdot 10^{6}\right.$ cells $)$ 
simulations on the refined grids, the general evolution stays unaffected by the improved resolution. This indicates that the flow is sufficiently well resolved on the coarser grid.

\section{CONCLUDING REMARKS}

The same models for real-gas thermodynamic effects, based on a volumecorrected version of the PR EoS, have been implemented into two LES codes, the density-based INCA and the pressure-based OpenFOAM. For the latter, both the pressure equation and the PISO solution algorithm have been modified to allow for a consistent pressure--velocity coupling with nonlinear EoSs. The new solvers have been used to simulate trans- and supercritical nitrogen injection into a warm surrounding. The computational results are in good agreement with the available experimental data of Mayer et al. [3], in particular, the location of breakup and the density decrease at supercritical injection conditions is well captured. At transcritical injection conditions, deviations could be observed in the transitional region of the jet, where the steep density decrease could not be reproduced to its full extent. It is remarkable that the results of both codes, INCA and OpenFOAM, are nearly identical, especially in the light of differing code architecture, inflow boundary condition, mesh and SGS turbulence modeling. In general, both solvers demonstrated their capability of predicting single-species, inert flows in at trans- and supercritical conditions. Future work will concentrate on extending the solver capabilities to multispecies and reacting flows.

\section{ACKNOWLEDGMENTS}

Financial support has been provided by the German Research Foundation (DFG - Deutsche Forschungsgemeinschaft) in the framework of the Sonderforschungsbereich Transregio 40. Computational resources have been provided by the Leibniz-Rechenzentrum München (LRZ) under grant h0983.

\section{REFERENCES}

1. Oschwald, M., J. J. Smith, R. Branam, J. Hussong, and A. Schik. 2006. Injection of fluids into supercritical environments. Combust. Sci. Technol. 178:49-100.

2. Telaar, J., G. Schneider, J. Hussong, and W. Mayer. 2001. Cryogenic jet injection: Test case RCM 1. 2nd Workshop (International) on Rocket Combustion Modeling Proceedings. Lampoldshausen, Germany. 
3. Mayer, W., J. Telaar, R. Branam, G. Schneider, and J. Hussong. 2003. Raman measurements of cryogenic injection at supercritical pressure. Int. J. Heat Mass Transfer 39:709-719.

4. Oschwald, M., A. Schik, M. Klar, and W. Mayer. 1999. Investigation of coaxial $\mathrm{LN}_{2} / \mathrm{GH}_{2}$-injection at supercritical pressure by spontaneous raman scattering. 35th AIAA/ASME/SAE/ASEE Joint Propulsion Conference. No. 99-2887.

5. Singla, G., P. Scouflaire, C. Rolon, and S. Candel. 2006. Planar laser-induced fluorescence of $\mathrm{OH}$ in high-pressure cryogenic $\mathrm{LOx} / \mathrm{GH}_{2}$ jet flames. Combust. Flame 144:151-169.

6. Mayer, W., and H. Tamura. 1996. Propellant injection in a liquid oxygen/gaseous hydrogen rocket engine. J. Propul. Power 12(6):1137-1147.

7. Chehroudi, B. 2012. Recent experimental efforts on high-pressure supercritical injection for liquid rockets and their implications. Int. J. Aerosp. Eng. ID 121802. $31 \mathrm{p}$.

8. Miller, R., K. Harstad, and J. Bellan. 2001. Direct numerical simulations of supercritical fluid mixing layers applied to heptane-nitrogen. J. Fluid Mech. 436:1-39.

9. Okong'o, N., and J. Bellan. 2003. Perturbation and initial reynolds number effects on transition attainment of supercritical, binary, temporal mixing layers. Comput. Fluids 33:1023-1046.

10. Taskinoglu, E., and J. Bellan. 2003. A posteriori study using a DNS database describing fluid disintegration and binary-species mixing under supercritical pressure: Heptane and nitrogen. J. Fluid Mech. 645:211-254.

11. Zong, N., and V. Yang. 2006. Cryogenic fluid jets and mixing layers in transcritical and supercritical environments. Combust. Sci. Technol. 178:193-227.

12. Oefelein, J. 2006. Mixing and combustion of cryogenic oxygen-hydrogen shearcoaxial jet flames at supercritical pressure. Combust. Sci. Technol. 178:229-252.

13. Matsuyama, S., J. Shinjo, S. Ogawa, and Y. Mizobuchi. 2010. Large eddy simulation of $\mathrm{LOx} / \mathrm{GH}_{2}$ shear-coaxial jet flame at supercritical pressure. 48th AIAA Aerospace Sciences Meeting including the New Horizons Forum and Aerospace Exposition.

14. Schmitt, T., J. Rodriguez, I. A. Leyva, and S. Candel. 2012. Experiments and numerical simulation of mixing under supercritical conditions. Phys. Fluids 24:055104.

15. Petit, X., G. Ribert, and P. Domingo. 2012. Large eddy simulation of supercritical fluid injection. AIAA Paper No. 2012-1268.

16. Park, T.S. 2012. LES and RANS simulations of cryogenic liquid nitrogen jets. J. Supercrit. Fluids 72:232-247.

17. Jarczyk, M., and M. Pfitzner. 2012. Large eddy simulation of supercritical nitrogen jets. AIAA Paper No. 2012-1270.

18. Jarczyk, M. 2013. Numerische Modellierung von turbulenten Strцmungen realer Gasgemische. PhD Thesis.

19. Niedermeier, C. A., H. Müller, M. Jarczyk, S. Hickel, N. A. Adams, and M. Pfitzner. 2013. Large-eddy simulation of turbulent trans- and supercritical mixing. AIAA Paper No. 2013-2950. 
20. Peng, D.-Y., and D. P. Robinson. 1976. A new two-constant equation of state. Ind. Eng. Chem. Fundam. 15(1):59-64.

21. Okong'o, N., K. Harstad, and J. Bellan. 2002. Direct numerical simulations of $\mathrm{O}_{2} / \mathrm{H}_{2}$ temporal mixing layers under supercritical conditions. AIAA J. 40(5):914926.

22. Jarczyk, M., M. Pfitzner, C. A. Niedermeier, S. Hickel, and N. A. Adams. 2011. Large eddy simulation of supercritical mixing layers. 4th European Conference for Aerospace Sciences (EUCASS). St. Petersburg.

23. Matheis, J., H. Müller, M. Pfitzner, and S. Hickel. 2016. Volume translation methods for real-gas computational fluid dynamics simulations. J. Supercrit. Fluid.107:422-432. doi:10.1016/j.supflu.2015.10.004.

24. Harstad, K., R. Miller, and J. Bellan. 1997. Effcient high-pressure state equations. AIChE J. 43(6):1605-1610.

25. Poling, B. E., J. M. Prausnitz, and J. P. O'Connell. 2000. The properties of gases and liquids. 5th ed. Mcgraw-Hill Professional. 803 p.

26. Goos, E., A. Burcat, and B. Ruscic. 2009. Extended third millennium ideal gas and condensed phase thermochemical database for combustion with updates from active thermochemical tables. Available at: http://burcat.technion.ac.il/dir (accessed October 26, 2015).

27. Chung, T.-H., M. Ajlan, L. L. Lee, and K. E. Starling. 1988. Generalized multiparameter correlation for nonpolar and polar fluid transport properties. Ind. Eng. Chem. Res. 27(4):671-679.

28. Hickel, S., N. A. Adams, and J. A. Domaradzki. 2006. An adaptive local deconvolution method for implicit LES. J. Comput. Phys. 213:413-436.

29. Hickel, S., and J. Larsson. 2009. On implicit turbulence modeling for LES of compressible flows. Advances in turbulence XII. Ed. E. Bruno. Springer proceedings in physics ser. Springer. 132:873-876. doi: 10.1007/978-3-642-03085-7_209.

30. Hickel, S., C. P. Egerer, and J. Larsson. 2014. Subgrid-scale modeling for implicit large eddy simulation of compressible flows and shock-turbulence interaction. Phys. Fluids 26:106101.

31. Smagorinsky, J. 1963. General circulation experiments with the primitive equations. Mon. Wea. Rev. 91:99-164.

32. Issa, R. 1985. Solution of the implicitly discretised fluid flow equations by operatorsplitting. J. Comput. Phys. 62:40-65.

33. Issa, R., B. Ahmadi-Befrui, K. Beshay, and A. Grosman. 1991. Solution of the implicitly discretised reacting flow equations by operator splitting. J. Comput. Phys. 93:388-410.

34. NIST. Thermophysical properties of fluid systems. Available at: http://webbook. nist.gov/chemistry/fluid (accessed October 26, 2015).

35. Soave, G. 1972. Equilibrium constants from a modified Redlich-Kwong equation of state. Chem. Eng. Sci. 27:1197-1203.

36. Branam, R., and W. Mayer. 2003. Characterization of cryogenic injection at supercritical pressure. J. Propul. Power 19(3):342-355. 
37. Nikuradse, J. 1966. Laws of turbulent flow in smooth pipes. NASA TT F-10.

38. Keppeler, R., J. Kranawetvogl, M. Jarczyk, and M. Pfitzner. 2011. Large eddy simulation von Rohr- Kanal- und Freistrahlströmung mit OpenFOAM. Technical Report. Available at: http://www.unibw.de/thermo/downloads/berichte (accessed October 26, 2015).

39. Schmitt, T., L. Selle, B. Cuenot, and T. Poinsot. 2009. Large-eddy simulation of transcritical flows. C. R. Mecanique 337:528-538.

40. Zong, N., H. Meng, S.-Y. Hsieh, and V. Yang. 2004. A numerical study of cryogenic fluid injection and mixing under supercritical conditions. Phys. Fluids 16(12):42484261.

41. Banuti, D. T., and K. Hannemann. 2010. Effect of injector wall heat flux on cryogenic injection. AIAA Paper No. 2010-7139. 